\title{
Historical ethnobotanical review of wild edible plants of Estonia (1770s-1960s)
}

\author{
Raivo Kalle', Renata Sõukand ${ }^{2 *}$ \\ ${ }^{1}$ Institute of Veterinary Medicine and Animal Sciences, Estonian University of Life Sciences, Kreutzwaldi 62, 51014 Tartu, Estonia \\ ${ }^{2}$ Estonian Literary Museum, Vanemuise 42, 51003 Tartu, Estonia
}

\begin{abstract}
This paper is a historical ethnobotanical review of wild plants used by the residents of present day Estonia during the 1770s1960s. Twenty two sources addressing historical ethnographical accounts of the use of wild food plants were analysed. The use of 149 taxa of vascular plants (over 6\% of Estonian vascular flora) and two lichens has been recorded. Although the data does not allow for reliable determination of the frequency of use of specific taxa among the population, general conclusions on the preferences for specific dishes made of wild food plants can be made. While the category of snacks covers the largest proportion of species used, a substantial addition to food rations was provided by bread ingredients (used predominantly in famine times), green vegetables used for making soup, and later jams and other dishes of wild berries. Also beverages (tea and coffee substitutes), beer and beer-like drinks were widely made, and the saps of several tree species were consumed in fresh and fermented form. The most important species, according to the criterion of diversity of use, were Carum carvi, Urtica dioica, and the wild berries Vaccinium vitis-idaea and Vaccinium myrtillus.
\end{abstract}

Keywords: wild food plants, historical ethnobotany, wild snacks, food culture, ethnographic and folkloristic records, wild edible plants

\section{Introduction}

In the quite recent past, during times of war, crop failure or other cases of food shortage, wild food plants have made a substantial addition to the human diet $[1,2]$. Nowadays, for the vast majority of the population of modern urbanized Europe, wild food plants seem to be of secondary importance as a resource for human nutrition; their gathering requires much more human labour than cultivated crops and vegetables do, their habitats are far from human settlements, and due to the loss of contact with nature, people simply do not know (or cannot recognize) wild plants. Nevertheless, even in Europe there are still many rural locations, where wild food plants are traditionally used on a daily basis. Such locations in southern and Western Europe are much appreciated by researchers in the field of ethnobotany [3-13]. In Northern Europe the research seems to be completed and it seems that not much can be added to it, as in countries with high industrialization and safe social settings the need for wild food is close to zero and the existing ethnographic resources are already thoroughly researched (for a few examples see [14-16]).

*Corresponding author. Email: renata@folklore.ee

This is an Open Access digital version of the article distributed under the terms of the Creative Commons Attribution 3.0 License (creativecommons.org/licenses/by/3.0/), which permits redistribution, commercial and non-commercial, provided that the article is properly cited.
A different situation exists in Eastern Europe within the post-socialist countries. The relatively recent open access to western goods still enables us to remember the use of wild plants on a national level, and research in these areas often gives fruitful results $[17,18]$. Moreover, some of these countries, like Estonia and its neighbours, have extensive ethnographic collections. While in some of the countries, such as Poland [19-21], such resources have been thoroughly analysed, the content of the others is still unknown to the rest of the world, as they are either not used after collection, or their analyses are published in the native languages only. This makes cross-cultural and geographical analysis difficult or even impossible [21].

To contribute a small stroke to the pattern of the use of wild plants of Europe from the Estonian side, we need first to review the historical use of wild food plants. The written legacy is rather thin: the few publications in German touching on the use of local species for food in the territory of present-day Estonia were written by Baltic German botanophiles [22-29]. Moreover, they often described Estonia and Livonia [22,23] together, some even including Courland $[27,28]$, and they cover only the period until the mid-19th century. Subsequent times are covered by the memory of respondents to folkloristic and ethnographic surveys, and later discussed by a few enthusiasts in Estonian only - dominantly through popular science publications. Hence, the aim of the study is to critically review all the publications concerning wild edibles covering the period 1770s-1960s. The data collected after 1970 has not yet been reflected in any reliable written source, except for a few of the authors' publications. 


\section{Material and Methods}

\section{Estonia - ecological and historical background}

Estonia belongs to the boreo-nemoral vegetation zone, its vegetation is very diverse. Forests, mires and grasslands alternate with cultivated land; meadows and grasslands constitute up to one fifth of it, whereas almost half of the territory is covered with forests, including one third of the area covered by peaty soils, with mixed forest dominating [30-33]. The number of known native and naturalized vascular plant species (with micro-species) of Estonia is estimated at 2200 [34,35].

Present-day Estonia covers an area of 45 thousand $\mathrm{km}^{2}$ with a population of 1.3 million inhabitants. Within the researched period, until 1918, Estonia was divided between two provinces of Russia (Estonia and Livonia); part of the latter now belongs to Latvia. Also a small area of the territory of present Estonia (Narva and its surroundings) belonged to the St. Petersburg province [36].

\section{Definition of wild food plants}

In the context of this article, the term "wild food plants" covers the popular category of wild plants in Estonia, referring mainly to plants growing without intended cultivation. It includes predominantly native and naturalized species, but also cultivars, if the sources suggest parts that are not usually eaten (like Secale creale L. and Linum usitatissimum L.), or plants, which are cultivated for non-food purposes (like Aesculus hippocastanum L.). Some of the species grow in the wild as well as in cultivated settings (like Armoracia rusticana Gaertn. et al. and Ribes nigrum L.).

\section{The sources of the review}

The documentation of traditions of plant use in Estonia began along with the start of documentation of Estonian flora [22], published by Baltic German Estophile, Pastor August Wilhelm Hupel (1737-1819). Although the list of plants was most probably compiled by the student of Carl Linneaus, Jakob Benjamn Fisher (1731-1793) [34], additions on the use and local names were made by Hupel. Hupel's later work also contained some relevant data [23].

Another important figure in Estonian ethnobotany was a German doctor residing on Island Ösel, Johann Wilhelm Ludvig von Luce (1756-1842). He not only wrote an ethnographic overview of the use of plants growing on his home island [24], but also educated peasants on how to survive the times of famine by relying on wild plants [25]. In the same period there was one more attempt to popularise the use of the plants, by another Baltic German Estophile Pastor Johann Heinrich Rosenplänter (1782-1846), but this book is still a manuscript [29]. For more details on the three aforementioned figures and their impact on Estonian ethnobotany follow Kalle and Sõukand [37].

Although there were a few more pieces of information here and there [26-28,38], gathered until the beginning of the 20th century, they rather repeat previous authors and do not differentiate suggetions and ethnographic use reports.

The later publications could be divided into two categories: a critical review of previous authors, and folklore and ethnographical data, including published collections of folklore texts.

The oldest ethnographical data on the use of wild food plants originate from the last decades of the 19th century. However, the systematic collection of relevant information started in the 1930s, when the Estonian National Museum and Estonian Folklore Archives published several questionnaires (e.g. [39-42]), addressing, among other subjects, the use of wild food plants. As the purpose of those collections was to save "antiquity", the data was left untouched until "better times".

The first researcher to analyse responses to the questionnaires from 1930 was ethnographer Aliise Moora (1900-1992). She published her short overviews in the then only popular natural science journal, Eesti Loodus [43-46]; also her monograph on the food of the peasants [47] contains some bits on the use of wild edible plants. Some pieces of this archival data, unpublished elsewhere, were also used in the authors' recent historical overview article in Estonian [48].

Nevertheless, the greatest ever collector and writer of the legacy of wild food plants was a doctor of botany, and for most of his life just a simple schoolteacher, Gustav Vilbaste (1885-1967). He collected his data on his own, mostly in 1920s-1930s, but some also later, until the 1960s. His profound analyses of the use of local plants contain not only his own data, but also critical reviews of earlier publications $[49,50]$. Still his most important work on wild edible plants is only a manuscript [51]. Another work, a monograph on the local plant names [52], was published almost 30 years after his death. Kalle and Sõukand [53] provide more information on Vilbaste's ethnobotanical impact.

We also use two sources publishing plain, uncommented folklore and ethnographic texts. The first of them, providing a few excerpts on edible wild plants within the framework of farm-life, was published before WW II [54]. The latter publication is an electronic database, containing predominantly data on the medicinal use of the plants, with a few pieces on wild edibles [55]; from it, only records collected before 1970 were evaluated. There are also two publications based on this database [56,57], but only the one published by Sõukand and Kalle [57] has been included in the present review, as the other deals, exceptionally, with medicinal plants. Moreover, as the article on "teetaimed" [57] is based on HERBA database [55], then if the plant was used only for making infusions and no additional data on other uses was available in the database, the published article became the only source in Tab. 1 .

Altogether, 22 publications were used for this review. Nevertheless, as some of the publications rely on the same sources or repeat previous statements, and all the results are obtained using different methodologies, it is not reasonable to count the importance of the species based on the number of citations.

\section{Methodology of plant identification}

All accessible sources addressing historical ethnographical accounts of the use of wild food plants were analysed. Historical sources written by Baltic Germans and Gustav Vilbaste contained predominantly Latin names of the plants. In the few cases where Gustav Vilbaste had over-identified the species listed by Baltic Germans, the identification was critically evaluated and Vilbaste's identification was considered correct (for example Urtica urens L. was changed to Urtica dioica L., Arctium lappa L. to Artium tomentosum Mill.). In the European context the last change may seem unreasonable, since $A$. lappa is a widely eaten species all over Europe. Nevertheless, in Estonia A. lappa could be found in only a few places on the coastline, while the uses originate from south Estonia, where A. lappa does not grow [58].

The written resources based on folklore and ethnographic collections [43-47,54,55] were critically evaluated and plant 
Tab. 1 List of wild food plants used in Estonia in 1770s-1960s.

\begin{tabular}{|c|c|c|c|c|c|}
\hline Family & Latin name & Local name & Parts used & Mode of use & Citing source \\
\hline Aceraceae & Acer platanoides $\mathrm{L}$. & Vaher & $\begin{array}{l}\text { sap, flowers, flower } \\
\text { buds }\end{array}$ & $\begin{array}{l}\text { drink (fresh and rarely fermented), } \\
\text { snacks, infusions, for making food } \\
\text { instead of water }\end{array}$ & {$[22,24,55,57]$} \\
\hline \multirow[t]{4}{*}{ Alliaceae } & Allium oleraceum $\mathrm{L}$. & Metslook & leaves, shoots, bulbs & spices & {$[25]$} \\
\hline & Allium schoenoprasum $\mathrm{L}$. & Murulauk, leemlaugud & leaves, bulbs & snacks, spices, soup & {$[24,48,52,55]$} \\
\hline & Allium spp. & Lauk & leaves & snacks, salad & {$[48]$} \\
\hline & Allium ursinum $\mathrm{L}$. & Karulauk & leaves & salad, snacks & {$[23,48]$} \\
\hline \multirow[t]{2}{*}{ Araceae } & Acorus calamus $\mathrm{L}$. & Kalmus & rhizomes* & unspecified food ${ }^{*}$, snacks & {$[23,55]$} \\
\hline & Calla palustris $\mathrm{L}$. & Soovõhk & rhizomes* & cooked for food ${ }^{*}$ & {$[28,51]$} \\
\hline \multirow[t]{18}{*}{ Asteraceae } & Achillea millefolium $\mathrm{L}$. & Raudrohi, verihein & $\begin{array}{l}\text { aerial part, flowers, } \\
\text { stalks }\end{array}$ & infusions, additive to beer & {$[47,55,57]$} \\
\hline & Antennaria dioica (L.) & Kassikäpp & aerial part & infusions & {$[57]$} \\
\hline & Gaertn. & & & & \\
\hline & Anthemis tinctoria L. & Rumaska & flowers & infusions & {$[57]$} \\
\hline & $\begin{array}{l}\text { Arctium tomentosum } \\
\text { Mill. }\end{array}$ & Kobruleht, takjas & $\begin{array}{l}\text { leaves, peeled young } \\
\text { stalks }\end{array}$ & soup & {$[22,23,45]$} \\
\hline & Artemisia absinthium L. & Koirohi, pänül & aerial part & additive to beer, beer-like drinks & {$[27,47,48,55]$} \\
\hline & $\begin{array}{l}\text { Centaurium erythraea } \\
\text { Rafn }\end{array}$ & Maasapp, põldhumalad & aerial part & additive to beer & $\begin{array}{l}{[22,24,47,} \\
50,52]\end{array}$ \\
\hline & Cichorium intubus L. & Sigur & roots & coffee substitute & {$[55]$} \\
\hline & Cirsium arvense (L.) & Ohakas, ohtja & young shoots & soup, bread ingredient & {$[22,23,45$} \\
\hline & $\begin{array}{l}\text { Scop. var. mite (Wimm. } \\
\text { et Grab.) Lange }\end{array}$ & & & & $51,55]$ \\
\hline & $\begin{array}{l}\text { Matricaria chamomilla } \\
\text { L. }\end{array}$ & Kummelid & flowers & infusions & [29] \\
\hline & Matricaria spp. & Kummel & flowers, aerial part & infusions & {$[57]$} \\
\hline & Scorzonera humilis L. & Mustjuur & $\begin{array}{l}\text { brown dust found in } \\
\text { flowers }\end{array}$ & snacks & {$[52]$} \\
\hline & Sonchus spp. & Piimohakas, ohtja & aerial part & soup, snacks & {$[22,29,51,55]$} \\
\hline & Tanacetum vulgare $\mathrm{L}$. & Reinvars & aerial part & beer additive & {$[47]$} \\
\hline & $\begin{array}{l}\text { Taraxacum officinale } \\
\text { Webb }\end{array}$ & Võilill, seaõitsed & leaves, flowers, roots & salad, snacks, coffee substitute, soup ${ }^{*}$ & {$[25,27,45,55]$} \\
\hline & Tragopogon pratensis $\mathrm{L}$. & Kohv, piimhain & young stalks & snacks & {$[52,55]$} \\
\hline & Tussilago farfara $\mathrm{L}$. & Paiseleht & leaves & soup & {$[28,45]$} \\
\hline Berberidaceae & Berberis vulgaris $\mathrm{L}$. & Kukerpuu, paburits & fruits, leaves & $\begin{array}{l}\text { snacks, infusions, jam, spices for } \\
\text { fermented cucumbers, additive to } \\
\text { deserts, wine }\end{array}$ & $\begin{array}{l}{[22,28,48,} \\
55,57]\end{array}$ \\
\hline \multirow[t]{2}{*}{ Betulaceae } & Alnus spp. & Lepp & $\begin{array}{l}\text { cambium, wood, } \\
\text { catkins }\end{array}$ & $\begin{array}{l}\text { snacks, for smoking meat, bread } \\
\text { ingredient }\end{array}$ & $\begin{array}{l}{[45,47,48} \\
54,55]\end{array}$ \\
\hline & Betula spp. & Kask, kõiv & $\begin{array}{l}\text { sap, leaves, catkins, } \\
\text { cambium, bark, } \\
\text { sawdust }\end{array}$ & $\begin{array}{l}\text { drink (fresh and fermented), snacks, } \\
\text { infusions, bread ingredient, salad, vinegar }\end{array}$ & $\begin{array}{l}{[22,24,44,45} \\
47,54,55,57]\end{array}$ \\
\hline \multirow[t]{2}{*}{ Boraginaceae } & Anchusa officinalis L. & Mesilill, imikas & nectar, young shoots ${ }^{*}$ & snacks, soup ${ }^{*}$, salad ${ }^{*}$ & {$[28,29,52]$} \\
\hline & Pulmonaria officinalis $\mathrm{L}$. & Imikas, söögilill & nectar & snacks & {$[52,55]$} \\
\hline \multirow[t]{9}{*}{ Brassicaceae } & $\begin{array}{l}\text { Alliaria petiolata (M. } \\
\text { Bieb.) Cavara \& Grande }\end{array}$ & Salukõdrik & aerial part* & unspecified food ${ }^{*}$ & {$[22]$} \\
\hline & $\begin{array}{l}\text { Armoracia rusticana } \\
\text { P. Gaertn., B. Mey. \& } \\
\text { Scherb. }\end{array}$ & Mädarõigas, mäerõigas & roots, leaves & $\begin{array}{l}\text { spices for fermented cucumber, additive } \\
\text { to food, meat dishes }\end{array}$ & {$[48,55]$} \\
\hline & Bunias orientalis $\mathrm{L}$. & Tölk, rakvere raibe & $\begin{array}{l}\text { young leaves, (peeled) } \\
\text { stalks }\end{array}$ & snacks, soup & {$[51]$} \\
\hline & $\begin{array}{l}\text { Capsella bursa-pastoris } \\
\text { (L.) Medik. }\end{array}$ & Hiirekõrv & aerial part & infusions & {$[57]$} \\
\hline & Cardamine amara $\mathrm{L}$. & Salat, kressid & leaves & salad & {$[49,52,55]$} \\
\hline & Cardamine pratensis $\mathrm{L}$. & Salat, kressid & leaves & salad & {$[49]$} \\
\hline & Crambe maritima $\mathrm{L}$. & Merekapstad & leaves ${ }^{*}$ & soup $^{*}$ & {$[25]$} \\
\hline & Sinapis arvensis $\mathrm{L}$. & Telg, tõlk & seeds, shoots & mustard, soup & {$[23,24,45,55]$} \\
\hline & Thlaspi arvense $\mathrm{L}$. & Litterhein & seeds & spices for soup and blood sausage & {$[55]$} \\
\hline
\end{tabular}


Tab. 1 (continued)

\begin{tabular}{|c|c|c|c|c|c|}
\hline Family & Latin name & Local name & Parts used & Mode of use & Citing source \\
\hline Cannabaceae & Humulus lupulus L. & Humal & fruits, young shoots & $\begin{array}{l}\text { additive to beer, beer-like drinks, bread } \\
\text { ingredient, soup }{ }^{*}, \text { salad }^{*}\end{array}$ & $\begin{array}{l}{[22,24,27,45} \\
47,48]\end{array}$ \\
\hline Caprifoliaceae & Lonicera xylosteum L. & $\begin{array}{l}\text { Kukepuu, ukekuusmaa, } \\
\text { sadakordne }\end{array}$ & fruits & snack & {$[22,24]$} \\
\hline \multirow[t]{3}{*}{ Chenopodiaceae } & Atriplex spp. & Malts & young shoots & soup & {$[25,55]$} \\
\hline & Chenopodium album $\mathrm{L}$. & Malts, hanemalts & young shoots, seeds & soup, bread ingredient & $\begin{array}{l}{[29,45,51} \\
52,55]\end{array}$ \\
\hline & Viburnum opulus L. & Lodjapuu, koera õidpuu & fruits & snacks, bread ingredient, jam & {$[24,48,55]$} \\
\hline Cladoniaceae & Cladonia spp. & $\begin{array}{l}\text { Valge sammal, sammal } \\
\text { (?) }\end{array}$ & thallus & bread ingredient & {$[38,45,47]$} \\
\hline Convallariaceae & $\begin{array}{l}\text { Maianthemum bifolium } \\
\text { (L.) F. W. Schmidt }\end{array}$ & $\begin{array}{l}\text { Viinamari, orava } \\
\text { marjad, metsviinamari }\end{array}$ & fruits & snacks & {$[29,52,55]$} \\
\hline Cornaceae & Cornus sanguinea L. & Komppuu, kontpuu & fruits & snacks & {$[55]$} \\
\hline Corylaceae & Corylus avellana $\mathrm{L}$. & Sarapuu, pähklipuu & nuts, catkins, buds & $\begin{array}{l}\text { snacks, desserts ingredient, bread } \\
\text { ingredient, seasonal beverage }\end{array}$ & $\begin{array}{l}{[24,27,28,45} \\
47,48,51,55]\end{array}$ \\
\hline \multirow[t]{2}{*}{ Crassulaceae } & $\begin{array}{l}\text { Jovibarba sobolifera } \\
\text { (Sims) Opiz (?) }\end{array}$ & Maarjasõnajalg & young leaves & soup & {$[55]$} \\
\hline & $\begin{array}{l}\text { Sedum maximum (L.) } \\
\text { Suter }\end{array}$ & Tuhkkartul & $\begin{array}{l}\text { radial tuber, leaves }{ }^{*} \text {, } \\
\text { young shoots* }\end{array}$ & cooked for snacks, soup ${ }^{\star}$, salad $^{*}$ & {$[28,52]$} \\
\hline Cupressaceae & Juniperus communis L. & Kadakas & cones, twigs, wood & $\begin{array}{l}\text { snacks, infusions, jam, drink, spices for } \\
\text { food, beer, beer-like drinks, smoking } \\
\text { meat, additive to fermented birch sap }\end{array}$ & $\begin{array}{l}{[22,24,28,43} \\
47,48,54,55 \\
57,62]\end{array}$ \\
\hline \multirow[t]{2}{*}{ Cyperaceae } & $\begin{array}{l}\text { Schoenoplectus } \\
\text { tabernaemontani (C. C. } \\
\text { Gmel.) Palla }\end{array}$ & Merekõrkjas & radial tuber & snacks & {$[54]$} \\
\hline & Unidentified Cyperaceae & $\begin{array}{l}\text { Villpea, sootutid, } \\
\text { soohein }\end{array}$ & stalks & snacks & {$[45]$} \\
\hline Dryopteridaceae & Dryopteridaceae (?) & Sõnajalg & leaves, roots & bread ingredient & {$[47,48,55]$} \\
\hline Equisetaceae & Equisetum arvense L. & $\begin{array}{l}\text { Kesatilgad, kitsenisad, } \\
\text { piibusk, seatilk, } \\
\text { savipähklid, }\end{array}$ & $\begin{array}{l}\text { spring shoots, radial } \\
\text { tuber }\end{array}$ & $\begin{array}{l}\text { soup, snacks (also cooked on fire), bread } \\
\text { ingredient }\end{array}$ & {$[45,51,55]$} \\
\hline \multirow[t]{9}{*}{ Ericaceae } & $\begin{array}{l}\text { Arctostaphylos uva-ursi } \\
\text { (L.) Spreng. }\end{array}$ & Leesikad, siapohl & leaves, fruits & infusions, snacks & {$[55,57]$} \\
\hline & $\begin{array}{l}\text { Calluna vulgaris (L.) } \\
\text { Hull. }\end{array}$ & Kanarbik & $\begin{array}{l}\text { flowers, aerial part, } \\
\text { seeds }\end{array}$ & infusions, bread ingredient & {$[45,55,57]$} \\
\hline & Empetrum nigrum $\mathrm{L}$. & $\begin{array}{l}\text { Kukesilmad, } \\
\text { varesemarja }\end{array}$ & fruits & snack, wine & {$[28,55]$} \\
\hline & Pyrola rotundifolia $\mathrm{L}$. & Talihaljak & leaves & infusions & {$[57]$} \\
\hline & $\begin{array}{l}\text { Rhododendron } \\
\text { tomentosum Harmaja }\end{array}$ & $\begin{array}{l}\text { Sookail, sookikas, } \\
\text { sookaer }\end{array}$ & aerial part & additive to beer, beer-like drinks & {$[22,47,48,55]$} \\
\hline & Vaccinium myrtillus L. & Mustikas & $\begin{array}{l}\text { fruits, leaves, flowers, } \\
\text { aerial part }\end{array}$ & $\begin{array}{l}\text { snacks, jam, soup, additive to desserts, } \\
\text { infusions }\end{array}$ & $\begin{array}{l}{[28,46,51,} \\
54,55]\end{array}$ \\
\hline & Vaccinium oxycoccos $\mathrm{L}$. & Kuremari, karbala & fruits & snacks, additive to dessert and sauerkraut & $\begin{array}{l}{[46,48,51} \\
54,55]\end{array}$ \\
\hline & Vaccinium uliginosum $\mathrm{L}$. & Sinikas & fruits & snacks, wine & {$[29,51,54,55]$} \\
\hline & Vaccinium vitis-idaea L. & Pohl, palohka, poolgas & $\begin{array}{l}\text { fruits, flowers, aerial } \\
\text { part }\end{array}$ & $\begin{array}{l}\text { snacks, jam, salad, additives to other } \\
\text { desserts, stored under water, infusions }\end{array}$ & $\begin{array}{l}{[28,29,46,48} \\
51,54,55,57]\end{array}$ \\
\hline \multirow[t]{5}{*}{ Fabaceae } & Lathyrus tuberosus L. & Seapähkel & radial tuber* & food, unspecified ${ }^{*}$ & {$[23]$} \\
\hline & Lotus corniculatus L. & Virapool & aerial part & infusions & {$[57]$} \\
\hline & Trifolium spp. & $\begin{array}{l}\text { Ristik, ristikhein, must } \\
\text { härjapea, valge härjapea }\end{array}$ & flowers & infusions & {$[55,57]$} \\
\hline & Vicia cracca L. & Kurehernes, hiirehernes & seeds & snacks & {$[29,49]$} \\
\hline & Vicia faba L. & Uba & stalks & additive to beer, for colouring & {$[47]$} \\
\hline Fagaceae & Quercus robur L. & Tamm & acorn, bark, leaves & $\begin{array}{l}\text { coffee substitute, spices for lacto- } \\
\text { fermented cucumbers, bread ingredient, } \\
\text { spices for beer-like drinks }\end{array}$ & $\begin{array}{l}{[24,27,28,45} \\
55,57]\end{array}$ \\
\hline Grossulariaceae & Ribes alpinum L. & $\begin{array}{l}\text { Mage sõstar, imalad, } \\
\text { mammuspuu, } \\
\text { naestõmari }\end{array}$ & fruits & snacks & {$[52]$} \\
\hline
\end{tabular}




\begin{tabular}{|c|c|c|c|c|c|}
\hline Family & Latin name & Local name & Parts used & Mode of use & Citing source \\
\hline & Ribes nigrum L. & Sitikas, must sõstar & fruits, leaves, twigs & $\begin{array}{l}\text { snacks, spices for lacto-fermented } \\
\text { cucumber, birch sap, infusions, bread } \\
\text { ingredient, species for beer-like drinks }\end{array}$ & $\begin{array}{l}{[45,47,48,55,} \\
57,62]\end{array}$ \\
\hline & Ribes uva-crispa L. & Kikerberi & leaves & infusions & {$[57]$} \\
\hline Hippocastanaceae & $\begin{array}{l}\text { Aesculus hippocastanum } \\
\text { L. }\end{array}$ & Kastan & seeds & coffee substitute, jam & {$[28,55]$} \\
\hline Hypericaceae & Hypericum spp. & $\begin{array}{l}\text { Naistepuna, viinalilled, } \\
\text { viinapunad }\end{array}$ & aerial part, flowers & $\begin{array}{l}\text { infusions, spices for blood sausages, } \\
\text { white pudding and vodka }\end{array}$ & {$[29,52,55,57]$} \\
\hline Hypolepidaceae & $\begin{array}{l}\text { Pteridium aquilinum } \\
\text { (L.) Kuhn }\end{array}$ & Sõnajalg & rhizomes & bread ingredient & {$[45,48,55]$} \\
\hline \multirow[t]{5}{*}{ Lamiaceae } & $\begin{array}{l}\text { Galeopsis tetrahit } \mathrm{L} \text {. } \\
\text { Lamium album } \mathrm{L} \text {. }\end{array}$ & $\begin{array}{l}\text { Mesililled, imikad } \\
\text { Iminõges, imikas, imik, } \\
\text { emanõges, naistenõges, } \\
\text { miiksmaasikad }\end{array}$ & $\begin{array}{l}\text { nectar } \\
\text { nectar, flowers, young } \\
\text { plants }\end{array}$ & $\begin{array}{l}\text { snacks } \\
\text { snacks, infusions, soup }\end{array}$ & $\begin{array}{l}{[29,52]} \\
{[28,45,52,55]}\end{array}$ \\
\hline & Mentha spp. & $\begin{array}{l}\text { Münt, vehverments, } \\
\text { piparmünt, põldmünt, } \\
\text { vesimünt }\end{array}$ & $\begin{array}{l}\text { aerial part, leaves, } \\
\text { flowers }\end{array}$ & $\begin{array}{l}\text { infusions, additive to food e.g. blood } \\
\text { sausages and white pudding, species for } \\
\text { vodka }\end{array}$ & {$[50,55,57]$} \\
\hline & Origanum vulgare L. & Pune, vorstirohi & $\begin{array}{l}\text { aerial part, leaves, } \\
\text { flowers }\end{array}$ & $\begin{array}{l}\text { infusions, spices for a variety of food, } \\
\text { blood sausages and white pudding, } \\
\text { additive to beer, soup }\end{array}$ & $\begin{array}{l}{[27,28,47,50} \\
55,57]\end{array}$ \\
\hline & Prunella vulgaris L. & Metspiparmünt & aerial part & infusions & {$[57]$} \\
\hline & Thymus serpyllum L. & Liivatee, viinapuna & $\begin{array}{l}\text { aerial part, flowers, } \\
\text { leaves }\end{array}$ & $\begin{array}{l}\text { infusions, spices for food, additive to } \\
\text { vodka }\end{array}$ & {$[28,50,55,57]$} \\
\hline Linaceae & Linum usitatissimum L. & Lina & capsule & bread ingredient & {$[45]$} \\
\hline Malvaceae & Malva pusilla Sm. & Kassirattad, kassinaerid & aerial part & soup $^{*}$, snacks & {$[25,55]$} \\
\hline Menyanthaceae & Menyanthes trifoliata L. & Ubaleht & aerial part & additive to beer & {$[24,28,48]$} \\
\hline Myricaceae & Myrica gale L. & Porss & leaves, twigs & additive to beer & {$[24,28,47,48]$} \\
\hline Nymphaeaceae & $\begin{array}{l}\text { Nymphaea candida C. } \\
\text { Presl }\end{array}$ & Vesiroos & rhizomes $^{*}$ & milled in food ${ }^{*}$ & {$[28,51]$} \\
\hline Oleaceae & Syringa vulgaris L. & Sirinäs & flowers & snacks for luck & {$[55]$} \\
\hline Onagraceae & $\begin{array}{l}\text { Epilobium angustifolium } \\
\text { L. }\end{array}$ & Kaporuski & young stalks*, leaves & soup $^{*}$, stew $^{*}$, infusions & {$[27,28,57]$} \\
\hline Orchidaceae & $\begin{array}{l}\text { Orchis morio L. } \\
\text { Platanthera bifolia (L.) } \\
\text { L. C. Rich. }\end{array}$ & $\begin{array}{l}\text { Jänese munad } \\
\text { Jänese munad }\end{array}$ & $\begin{array}{l}\text { tubers }^{*} \\
\text { tubers }^{*}\end{array}$ & $\begin{array}{l}\text { bread ingredient }{ }^{*}, \text { porridge }^{*} \\
\text { bread ingredient }\end{array}$ & $\begin{array}{l}{[25]} \\
{[25]}\end{array}$ \\
\hline Oxalidaceae & Oxalis acetosella $\mathrm{L}$. & $\begin{array}{l}\text { Jänese hapuoblikas, } \\
\text { kikikapsas, saksamaa } \\
\text { oblikad, jänese kapstad }\end{array}$ & leaves, flowers & snacks, soup & $\begin{array}{l}{[24,25,27,45} \\
51,54,55]\end{array}$ \\
\hline Papaveraceae & $\begin{array}{l}\text { Fumaria officinalis } \mathrm{L} \text {. } \\
\text { Papaver somniferum } \mathrm{L} \text {. }\end{array}$ & $\begin{array}{l}\text { Kolmekõrraline hain } \\
\text { Moonid }\end{array}$ & $\begin{array}{l}\text { aerial part } \\
\text { seeds }\end{array}$ & $\begin{array}{l}\text { infusions } \\
\text { snacks, additive on home-backed roll }\end{array}$ & $\begin{array}{l}{[57]} \\
{[55]}\end{array}$ \\
\hline Parmeliaceae & $\begin{array}{l}\text { Cetraria islandica (L.) } \\
\text { Ach. }\end{array}$ & $\begin{array}{l}\text { Nõmmesammal, } \\
\text { põdrasammal, islandi } \\
\text { sammal, liivasamblad, } \\
\text { sammal (?) }\end{array}$ & thallus & bread ingredient, infusions & $\begin{array}{l}{[25,38,45,47} \\
55,57]\end{array}$ \\
\hline \multirow[t]{2}{*}{ Pinaceae } & Picea abies (L.) H. Karst. & Kuusk & $\begin{array}{l}\text { shoots (young), } \\
\text { needles, cambium, } \\
\text { cones, resin }\end{array}$ & snacks, smoking of meat & {$[45,48,54,55]$} \\
\hline & Pinus sylvestris L. & Pedaja, mänd, pettäi & $\begin{array}{l}\text { shoots (young), } \\
\text { cambium, cones, bark }\end{array}$ & $\begin{array}{l}\text { snacks, bread ingredient, smoking of } \\
\text { meat }\end{array}$ & {$[45,47,48,55]$} \\
\hline Plantaginaceae & Plantago major L. & Teeleht & leaves & infusions, soup, salad ${ }^{\star}$ & $\begin{array}{l}{[22,27-29,48} \\
50,57]\end{array}$ \\
\hline \multirow[t]{4}{*}{ Poaceae } & Bromus secalinus L. & Lusted, kaer & seeds & distilled vodka, beer ingredient & {$[55]$} \\
\hline & $\begin{array}{l}\text { Phragmites australis } \\
\text { (Cav.) Trin. ex Steud. }\end{array}$ & Roog & roots & snacks & {$[48,54]$} \\
\hline & Poaceae & Aganad, kõrred & soft part of stalk, chaff & snacks, bread ingredient & {$[45,47,48]$} \\
\hline & Secale cereale $\mathrm{L}$. & Rukis & young crops & infusions & {$[57]$} \\
\hline Polygonaceae & $\begin{array}{l}\text { Polygonum amphibium } \\
\text { f. terrestre (Leers) S. F. } \\
\text { Blake }\end{array}$ & Seavinnal & roots & snacks & {$[55]$} \\
\hline
\end{tabular}




\begin{tabular}{|c|c|c|c|c|c|}
\hline Family & Latin name & Local name & Parts used & Mode of use & Citing source \\
\hline & Rumex acetosa $\mathrm{L}$. & Oblikas, hapuoblikas & leaves & snacks, soup, pie & $\begin{array}{l}{[23,25,29,45} \\
51,54,55]\end{array}$ \\
\hline & Rumex acetosella $\mathrm{L}$. & Hapuoblikas & leaves, young stalks & snacks & {$[24,52]$} \\
\hline Polypodiaceae & Polypodium vulgare L. & $\begin{array}{l}\text { Magusjuur, suhkrupuu, } \\
\text { lagrits }\end{array}$ & rhizomes & snacks & {$[55]$} \\
\hline \multirow[t]{3}{*}{ Primulaceae } & $\begin{array}{l}\text { Lysimachia nummularia } \\
\text { L. }\end{array}$ & Trudamorda & aerial part & infusions & {$[57]$} \\
\hline & Lysimachia vulgaris $\mathrm{L}$. & Metsvits & medulla* & snacks ${ }^{\star}$ & {$[27,50]$} \\
\hline & Primula veris $\mathrm{L}$. & Nurmenukk, käekaatsed & $\begin{array}{l}\text { flowers, leaves, steams, } \\
\text { pistil, aerial part, } \\
\text { nectar, flower stalks }\end{array}$ & $\begin{array}{l}\text { snacks, infusions, snacks for luck, wine }{ }^{*} \text {, } \\
\text { beer-like drinks }\end{array}$ & $\begin{array}{l}{[24,27,49,54} \\
55,57]\end{array}$ \\
\hline \multirow[t]{3}{*}{ Ranunculaceae } & Caltha palustris $\mathrm{L}$. & Varsakabi, konnakapsad & aerial part, buds* & bread ingredient, substitute for capers ${ }^{*}$ & {$[24,45,47,49]$} \\
\hline & Ranunculus ficaria L. & Kanakoole & young shoots, tubers & $\begin{array}{l}\text { salad (tubers were used only after soaking } \\
\text { in salted water) }\end{array}$ & {$[23,50,51]$} \\
\hline & $\begin{array}{l}\text { Thalictrum } \\
\text { aquilegiifolium } \mathrm{L} \text {. }\end{array}$ & $\begin{array}{l}\text { Ängelhein, kirnputk, } \\
\text { koerkuseputk, koerputk }\end{array}$ & flowers & infusions & {$[57]$} \\
\hline \multirow[t]{24}{*}{ Rosaceae } & $\begin{array}{l}\text { Alchemilla vulgaris auct. } \\
\text { (coll.) }\end{array}$ & Krooklehed, kortsleht & leaves & infusions & {$[55,57]$} \\
\hline & Crataegus spp. & $\begin{array}{l}\text { Leivamari, viirpuu, } \\
\text { leivapuu }\end{array}$ & fruits & snacks, bread ingredient & {$[48,55]$} \\
\hline & Filipendula ulmaria (L.) & Angervaks, viinarohi & flowers, aerial part & infusions, spices for vodka & {$[52,55,57]$} \\
\hline & Maxim. & & & & \\
\hline & $\begin{array}{l}\text { Filipendula vulgaris } \\
\text { Moench }\end{array}$ & Angerpist, viinarohi & $\begin{array}{l}\text { flowers, aerial part, } \\
\text { radial tuber* }\end{array}$ & $\begin{array}{l}\text { infusions, bread ingredient }{ }^{\star} \text {, spices for } \\
\text { vodka }\end{array}$ & {$[28,49,55,57]$} \\
\hline & Fragaria vesca L. & Maasikas, metsmaasikas & $\begin{array}{l}\text { fruits, leaves, flowers, } \\
\text { aerial part }\end{array}$ & snacks, jam, infusions, additive to dessert & $\begin{array}{l}{[24,27,29,46} \\
51,54,55,57]\end{array}$ \\
\hline & Fragaria viridis A. Duch. & $\begin{array}{l}\text { Muulukas, } \\
\text { mullikmaasikad }\end{array}$ & fruits & snacks, jam, additive to dessert & {$[49,51,55]$} \\
\hline & Geum rivale $\mathrm{L}$. & $\begin{array}{l}\text { Mesikupp, karukellad, } \\
\text { ärjamürakad, härjapea, } \\
\text { surnu sukapael }\end{array}$ & flowers, nectar, roots ${ }^{\star}$ & snacks, infusions, spices for food ${ }^{*}$ & {$[24,52,55,57]$} \\
\hline & Geum urbanum L. & Maamõõl & young shoots ${ }^{\star}$, roots & food $^{*}$, spices $^{*}$ & {$[24,27]$} \\
\hline & Malus domestica Borkh. & Õunapuu & fruits, leaves, wood & $\begin{array}{l}\text { snacks, wine, vinegar, infusions, additive } \\
\text { to sauerkraut, smoking of meat }\end{array}$ & {$[48,54,55,57]$} \\
\hline & Malus sylvestris Mill. & Metsõunapuu & fruits & snacks, wine & {$[24,29,48,51]$} \\
\hline & $\begin{array}{l}\text { Potentilla erecta (L.) } \\
\text { Raeusch }\end{array}$ & $\begin{array}{l}\text { Tedre madar, } \\
\text { tedremaran }\end{array}$ & rhizomes & cooked for food, additive to vodka & {$[29,45,48]$} \\
\hline & Prunus cerasus L. & Kirsipuu & fruits, leaves, flowers & $\begin{array}{l}\text { snacks, infusions, spices for fermenting } \\
\text { cucumbers }\end{array}$ & {$[55,57]$} \\
\hline & $\begin{array}{l}\text { Prunus domestica L. } \\
\text { subsp. insititia }\end{array}$ & Kreegipuu & leaves & infusions & {$[57]$} \\
\hline & Prunus domestica L. & Ploomipuu & flowers & infusions & {$[57]$} \\
\hline & Prunus padus L. & Toomingas & fruits, leaves, flowers & $\begin{array}{l}\text { snacks, spices for fermenting cucumbers, } \\
\text { snacks for luck }\end{array}$ & $\begin{array}{l}{[24,46,51} \\
54,55]\end{array}$ \\
\hline & Rosa spp. & Kibuvits, orjavits, roos & fruits, flowers & $\begin{array}{l}\text { snacks, infusions, jam, kissel, bread } \\
\text { ingredient, coffee, dessert }\end{array}$ & $\begin{array}{l}{[24,48,54} \\
55,57]\end{array}$ \\
\hline & Rubus caesius $\mathrm{L}$. & Põldmurakas, põldmari & fruits & snacks, wine, jam & $\begin{array}{l}{[28,49,52,} \\
54,55]\end{array}$ \\
\hline & Rubus chamaemorus L. & Murakas, käbalad & fruits, leaves, flowers & snacks, infusions, jam & $\begin{array}{l}{[28,29,51,54} \\
55,57]\end{array}$ \\
\hline & Rubus idaeus L. & Vaarikas, vabarn & fruits, leaves, twigs & $\begin{array}{l}\text { snacks, infusions, additive to fermented } \\
\text { cucumbers, jam, coffee substitute, wine }\end{array}$ & $\begin{array}{l}{[24,27,51,54} \\
55,57]\end{array}$ \\
\hline & Rubus saxatilis $\mathrm{L}$. & Lillakas & fruits & snacks, wine & {$[51,55]$} \\
\hline & Sorbus aucuparia L. & Pihlakas & fruits, flowers, sap & $\begin{array}{l}\text { snacks, jam, wine, infusions, bread } \\
\text { ingredient, kvass }\end{array}$ & $\begin{array}{l}{[27,44,46-48} \\
51,54,55]\end{array}$ \\
\hline & $\begin{array}{l}\text { Sorbus intermedia } \\
\text { (Ehrh.) Pers. }\end{array}$ & Leivamari, pooppuu & fruits & snacks, bread ingredient & {$[48,55]$} \\
\hline & Sorbus rupicola Hedl. & Kirsi-pooppuu & fruits & bread ingredient & {$[55]$} \\
\hline
\end{tabular}




\begin{tabular}{|c|c|c|c|c|c|}
\hline Family & Latin name & Local name & Parts used & Mode of use & Citing source \\
\hline \multirow[t]{2}{*}{ Salicaceae } & Populus tremula L. & Haab & wood, sawdust & $\begin{array}{l}\text { smoking of meat, bread ingredient, } \\
\text { vinegar }\end{array}$ & {$[47,48,55]$} \\
\hline & Salix spp. & Paju & leaves & bread ingredient & {$[45]$} \\
\hline \multirow[t]{2}{*}{ Scrophulariaceae } & Pinguicula vulgaris $\mathrm{L}$. & Võipätakas & aerial part & additive for souring the milk & {$[27,28,50]$} \\
\hline & Verbascum spp. & Üheksavägine & flowers & infusions & [57] \\
\hline Solanaceae & Solanum tuberosum $\mathrm{L}$. & Kartul & aerial part & soup & {$[54,55]$} \\
\hline Tiliaceae & Tilia spp. & Pärn, niin, pähnapuu & $\begin{array}{l}\text { flowers, buds, sap, } \\
\text { twigs and flower buds }\end{array}$ & infusions, snacks & {$[44,55,57]$} \\
\hline Typhaceae & Typha spp. & $\begin{array}{l}\text { Hunditõlv, hundipurik, } \\
\text { hundikurk }\end{array}$ & $\begin{array}{l}\text { rhizomes, young } \\
\text { shoots }^{*}\end{array}$ & snacks, salad ${ }^{*}$, stew $^{*}$ & {$[24,28,45,48]$} \\
\hline \multirow{5}{*}{ Umbelliferae } & Angelica sylvestris L. & $\begin{array}{l}\text { Pütski, heinputk, } \\
\text { söögiputk }\end{array}$ & $\begin{array}{l}\text { young shoots, peeled } \\
\text { stalks, aerial part }\end{array}$ & soup, snacks, infusions & $\begin{array}{l}{[45,49,51,54} \\
55,57]\end{array}$ \\
\hline & $\begin{array}{l}\text { Anthriscus sylvestris (L.) } \\
\text { Hoffm. }\end{array}$ & Pütski, penipütsk & $\begin{array}{l}\text { young shoots, peeled } \\
\text { stalks }\end{array}$ & soup, snacks & {$[45,49,51]$} \\
\hline & Carum carvi L. & Köömen, köömlid & seeds, leaves, stems & $\begin{array}{l}\text { spices for variety of food, bread, blood } \\
\text { sausages and white pudding, vodka, } \\
\text { sauerkraut, fermented cucumbers, birch } \\
\text { sap and other preserves, snacks, soup, } \\
\text { infusions, additive to beer-like drinks and } \\
\text { cottage cheese }\end{array}$ & $\begin{array}{l}{[22,23,25,29,} \\
47,48,51,55, \\
57,62]\end{array}$ \\
\hline & $\begin{array}{l}\text { Heracleum sphondylium } \\
\text { L. (?) }\end{array}$ & Karuputk, natid & $\begin{array}{l}\text { young shoots, peeled } \\
\text { stalks }\end{array}$ & soup, snacks & {$[22-24,27,48]$} \\
\hline & Pimpinella anisum L. & Ingver, maaingver & roots & spices for food & {$[55]$} \\
\hline Urticaceae & Urtica dioica L. & $\begin{array}{l}\text { Nõges, kõrvenõges, } \\
\text { raudnõges }\end{array}$ & $\begin{array}{l}\text { leaves, aerial part } \\
\text { (young), stalks, shoots, } \\
\text { roots }\end{array}$ & $\begin{array}{l}\text { soup, infusions, bread ingredient, for } \\
\text { smoking meat }\end{array}$ & $\begin{array}{l}{[22,23,25,26,} \\
45,48,51,54 \\
55,57]\end{array}$ \\
\hline Valerianaceae & Valeriana officinalis L. & Palderjan & $\begin{array}{l}\text { aerial part, flowers, } \\
\text { roots }\end{array}$ & infusions & {$[57]$} \\
\hline Violaceae & Viola odorata L. & Kannike & flowers & dessert, spices for syrup and vinegar ${ }^{*}$ & {$[28,55]$} \\
\hline
\end{tabular}

(?) - name did not have enough details for specific identification. ${ }^{*}$ Data of uncertain ethnic origin.

names identified through using local names [52]. Identification changes were made where necessary, relying on the work of Sõukand and Kalle [55]. For example identifications followed by Moora [45]: Dryopteris spp. to Pteridium aquilinum (L.) Kuhn. The previously unidentified species Sinapis arvensis L. was also added to the list based on its local name ("tõlk"). Based on the same principles two plants identified in sources relying on folklore were excluded: Angelica archangelica L. and Conium maculatum L. The latter was named kaljaputk, indicating its use for making kvass. Regardless of the fact that some nations do use deadly poisonous plants for food after certain preparations (e.g. [59]), the text claiming the use of kaljaputk did not contain any other reliable information, nor a description of its preparation allowing for its identification as C. maculatum.

If the local plant name listed in the sources did not allow for identification on the species level, a taxon was identified as a genus. The list of all species that could potentially be eaten within the particular genus is provided in Tab. 2. Nevertheless, two taxa listed in folklore and written sources did not allow for identification below the family (Cyperaceae and Poaceae), and in both families the softer part of the stalks of several species were eaten as snacks.

The texts in which plant identification credibility (discussed by Łuczaj [60]) was very low or the taxon was impossible to detect, were left aside.

\section{The reliability and completeness of the presented data}

Compared to Estonian serfs, the food selection of Baltic German landlords was much more diverse and contained many wild spices, used to make salads and soups; as well as numerous wild berries used to make juice, wine, salads and jams. Therefore, Baltic Germans were trying to educate local peasants through books and personal advice [22,25].

Memories of the earliest folklore texts reach back to the mid-19th century. Therefore they do not contain the specific species found in Hupel's and Luce's publications or later interpretations in Vilbaste's study [51] (see uses marked with “»” in Tab. 1). The original publications are written in a manner that does not enable us to understand where, or, more particularly, who used to make salad from the leaves of Taraxacum officinale 
Tab. 2 The species potentially named in texts where the taxa could be identified to the genus only. Species are presented in the order of assumed use frequency.

Genera Species potentially used

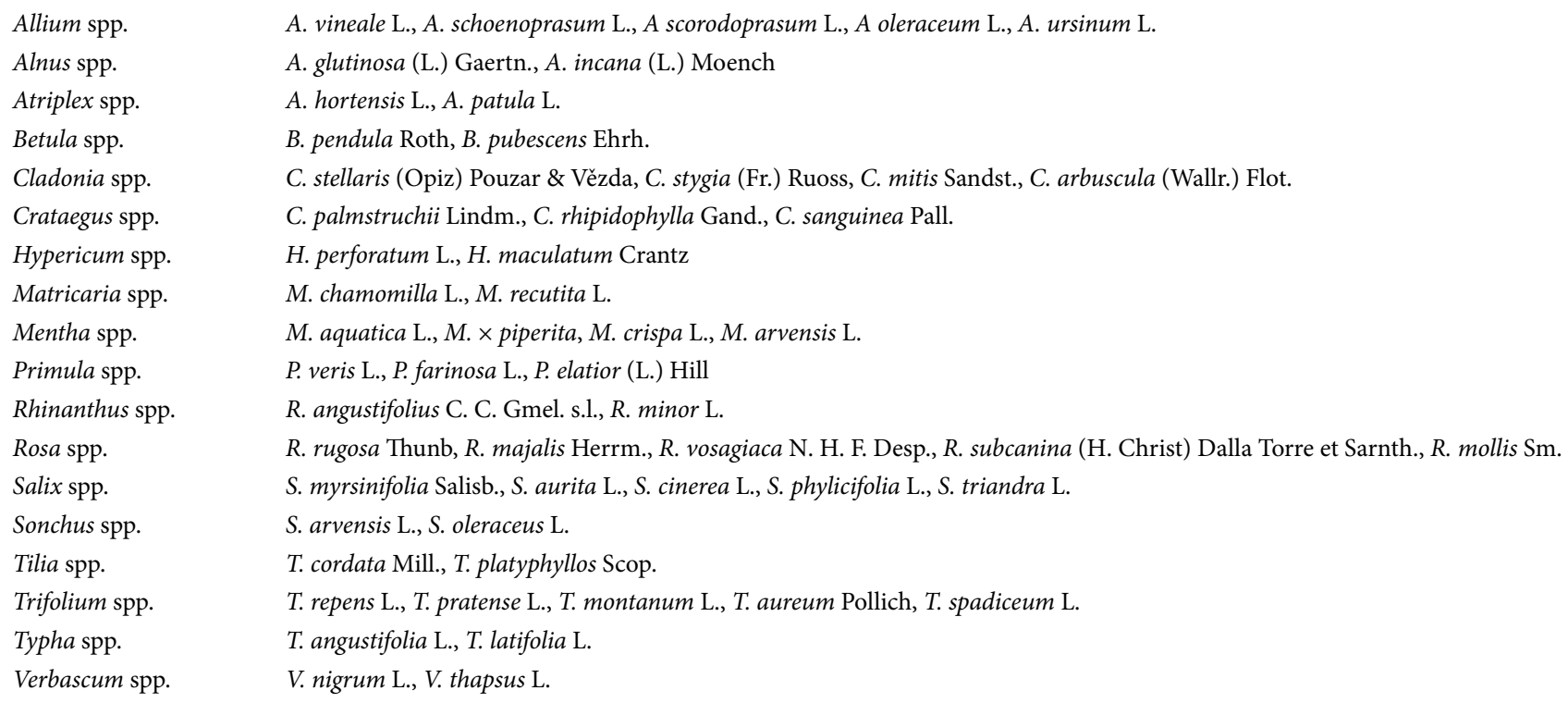

Allium spp.

Alnus spp.

Atriplex spp.

Betula spp.

Cladonia spp.

Crataegus spp.

Hypericum spp.

Matricaria spp.

Mentha spp.

Primula spp.

Rhinanthus spp.

Rosa spp.

Salix spp.

Sonchus spp.

Tilia spp.

Trifolium spp.

Typha spp.

Verbascum spp.

Webb, soup of leaves of Crambe maritima L. or peeled stems of Arctium tomentosum Mill.; who used to make various food from the rhizomes of Nymphaea candida C. Presl, Calla palustris L., Acorus calamus L., the tubers of Orchidaceae and Lathyrus tuberosus L. or the aerial parts of Alliaria petiolata (M. Bieb.) Cavara et Grande; or who used the buds of Caltha palustris L. as a substitute for capers, etc. The actual use of all those species by Estonian peasants cannot be confirmed, but cannot be disproved either. These species could have been used by local Baltic Germans, Swedes, or Russians, or on the territory of Livonia.

Although the majority of the cited authors use folkloristic and ethnographic sources, the whole existing archival data on the use of wild food plants in Estonia has never been digitized nor thoroughly systematized. Until this is done, it is impossible to state if any more taxa were historically used.

\section{Results and discussion}

During the research period, altogether 149 vascular plant taxa (approx. $6.6 \%$ of native and naturalized flora of Estonia) and 2 lichens were consumed (Tab. 1). If we exclude from the calculation those 19 species used for making infusions (recreational teas) only, there will be 130 taxa left, covering almost $5.8 \%$ of the Estonian vascular flora. All the taxa belong to 123 genera from 56 families [largest Rosaceae (22 taxa) and Asteraceae (16 taxa)]. The list includes 18 tree taxa, 15 shrubs, 11 subshrubs and one vine. Among the herbaceous plants 76 are perennials, 6 biennials and 5 annuals.

Most of the taxa were used in a variety of ways, utilizing various parts of the plant, only a few plants were used for one specific purpose (usually infusions or snacks only). The species that were used for infusions only had popularly recognized medicinal properties, and snacks were occasionally eaten raw. Among the most diversely consumed plants we can single out Carum carvi L., Urtica dioica L., and a variety of wild berries
(Vaccinium vitis-idaea L., Vaccinium myrtillus L., etc) and fruits (Sorbus aucuparia L., Rosa spp., etc).

\section{Snacks}

Seasonal snacks form the largest group of wild plants used, covering almost half of all the species (72). Most of the snacks (fruits, nectar, leaves, buds, cambium, stalks, etc.) were eaten in outdoor conditions without prior processing, while some were cooked [as tubers of Sedum maximum (L.) Suter and Equisetum arvense L.], frozen (such as fruits of Viburnum opulus L., Malus sylvestris Mill. and Sorbus aucuparia L.), or dried (Corylus avellana L. and Vaccinium myrtillus L.). While in earlier ethnobotanical research snacks were associated predominantly with children $[60,61]$, in 19th century Estonia snacks were eaten by both children and adults, although more recently snacks tended to be consumed by children. Still, specific plants have been used as children's snacks only, such as the rhizomes of Polypodium vulgare L (also used as medicine instead of Glycyrrhiza glabra L.), the dark "coffee" dust in the flowers of Scorzonera humilis L., a species used for sucking nectar, and a few others. Sweet snacks with sugar added were rather uncommon among peasants until the first decades of the 20th century due to the lack of sugar, but after that many berries were eaten with sugar and milk (later cream) added. Some other sweets were also made of flowers as candy, for example of Viola odorata L. and Rosa spp.

\section{Beverages}

Beverages made of wild plants are the second largest group of wild food consumed. As many as 54 vascular plants and one lichen were used to make infusions [57]. In the time of scarcity, the infusion made of well-tasting plants was especially appreciated as it gave better taste to the food. Twelve taxa were used for making or flavouring beer and eight for beer-like beverages. Several parts (acorns, roots, twigs) of six species were used to make coffee. Also saps of different trees were used (fresh or fermented), the most widespread being saps of Betula spp. and 
Acer platanoides L. (see also [62]). Fruits of only eight taxa were used for making wine; this practice started only at the beginning of the 20th century, when sugar became more accessible.

\section{Bread ingredients}

In the 18th and the first half of the 19th century the main foods for peasants were bread and other products made of flour [47]. Thus every crop failure caused by bad weather brought along large-scale famine [63]. This explains why a large group of vascular plants (30) and the thallus of two lichens were used as bread ingredients. A vast variety of plant parts was used for filling bread, including (dried) bulbs, leaves, shoots, cambium and bark. Some plants, especially powdered roots and young shoots, were added to the bread to "prolong" the flour; laced bread was called "näljaleib" (hunger-bread) in times of famine, and "hädaleib" (need-bread) before the new crop. Some of the ingredients that had pleasant taste were used also for flavouring bread in better times, for example Sorbus spp. fruits, also Carum carvi fruits were used, along with providing better taste to ensure longer preservation of the bread.

During the second half of the 19th century, when potato became widespread, the nutritional situation of peasants became better. The need for wild plants as bread ingredients gradually diminished, until it almost disappeared by the 1960s, partly also because, due to socio-economic changes, bread was then made at home by only a very few people. Still, few wild ingredients were later also used in making home-made rolls.

\section{Soups and gruel}

Greens (U. dioica, Rumex spp. and Cirsium spp., being most mentioned in folklore among them) that popped up after the snowmelt, were used for making a variety of dishes such as soup and porridge during food shortages. This happened almost every year to a greater or lesser extent, and the quantity of the added greens depended on the stocks of flour and grain available, but also on regional and household food habits. Altogether, 26 species were used in the form of soup, which argues against the statement of Baltic German authors that the monotonous diet of Estonians contained a very limited amount of green vegetables [22,25]. Moreover, some sources from the 18 th century complain that local peasants eat too many greens in the spring famines and die of it [63].

\section{Spices}

Altogether 18 taxa have been used for seasoning different foods, the majority of them very commonly. Eight taxa were used as ingredients for lacto-fermented cucumbers (mostly leaves, but also fruits, twigs and roots); three taxa were used for seasoning sauerkraut and another three for flavouring soured birch sap. Some of the herbs used for infusions were also used to season vodka (six taxa) and blood sausages (four taxa). Eight taxa were used as additives to beer. Seven taxa were used to smoke meat or fish; mostly wood, but also leaves and cones. Along with the seasoning purposes, spices were intended to improve the storing properties of the foods they were used in, for example a handful of Carum carvi in bread batter, a few segments of Armoracia rusticana root in cucumber preserves, a few inflorescences of Rhododendron tomentosum Harmaja. for a bucket of beer, etc.

\section{Fruits and seeds}

Of the fleshy fruits, 14 were fruits of wild shrubs and semi-shrubs and 11 were fruits of wild trees, all of them were consumed as snacks, but some were also used for making food, or as spices, and/or preserved for the winter in different ways. Until the end of 19th century the preservation of wild fruits was rather rare and mostly carried out in the form of drying or freezing, also under-water storage, and the fruits were used predominantly for healing purposes. Since the beginning of the 20th century, the wider making of jams (for food) began, reaching its peak in the1970s-1980s.

Although the majority of the seeds and dry fruits ( 7 species) were used as snacks only, there were also a few used for making food (nuts of Corylus avellana L. and acorns of Quercus robur L.). Also, the caryopses (grains) of Bromus secalinus L. were used to make vodka and beer; and seeds of Sinapis arvensis L. were used as the main component of mustard.

\section{Salads}

For many species, the green parts of the vegetable such as leaves and stalks were consumed. However, the use of wild green vegetables for making salad was rather limited, only 11 species were consumed. The use of salad seems to be a clear imitation of the landlords' food-habits. For example two local species Cardamine amara L. and Cardamine pratensis L. were named after Lepidium spp., cultivated and consumed in manors [49]. The use of the other greens for salad is of quite late origin (beginning of 20th century).

\section{Underground parts}

Underground parts of the plants were consumed rather rarely, being predominantly either famine food or snacks; altogether 24 taxa were used. Of them, 7 species with underground parts used were of uncertain ethnic origin (marked with " $\star$ " in Tab. 1; see "The reliability and completeness of the presented data" section for details). Underground parts were mostly used as bread ingredients, but also as snacks [Equisetum arvense L., Phragmites australis (Cav.) Trin. ex Steud., Polypodium vulgare L.] and cooked food [Potentilla erecta (L.) Raeusch, Sagittaria sagittifolia L.]. Roots of some taxa were also used as spices (Armoracia rusticana Gaertn. et al.), or for making infusions (Valeriana officinalis L.) or coffee (Taraxacum officinale).

\section{Alien plants, literature and education}

While the local habitants had a long time to acquire knowledge of the native plants, the quick introduction of alien plants into the diet of Estonians came about mostly through the popularizing literature. At the end of the 19th century, general literacy in Estonia was the highest in all of the Russian Empire, being almost $90 \%$ at the end of the 19th century [64]. The first alien taxon of cultivated ornamental plants introduced for food was Caragana arborescens Lam. [65], and although there are no records of its use during the period of the research, probably due to its very early publication date, its seeds and flowers were eaten later (unpublished fieldwork results). Nevertheless, the use of the conkers of Aesculus hippocastanum L. is clearly of literature origin, as its use for making coffee was widely propagated at the beginning of the 20th century (for example see $[66,67])$.

The use of native plants was also acquired through the literature, like the use of the thallus of Cetraria islandica (L.) Ach. as a bread ingredient from [25] and the teachings of local Baltic Germans. Estonian pioneer doctor and the author of national epic Friedrich Reinhold Kreutzwald (1803-1882) reports that flour made of soaked and dried thallus saved town citizens of Vorru from famine in the 1830s, as the town's mayor supported 
its use for making bread [38]. Another lichen taxon, Cladonia spp., was also used instead of $C$. islandica, as people often did not differentiate them on the name level [68].

Nevertheless, most of the teachings communicated through the literature were not accepted. For example the very popular first herbal written in Estonian by Baltic German pastor Otto August Jannau (1800-1865) teaches the use of Glyceria spp. for making manna porridge [69], but despite the fact that this plant was widely eaten in parts of central Europe [70], Estonian peasants did not adopt its use, probably because they already had potatoes and severe famines were only history at the time the book was published.

The practice of making of jam from wild berries most probably first came from the book by local schoolteacher and pomologist Jaan Spuhl-Rotalia (1859-1916), who taught the use of almost all wild berries growing in Estonia [66]. Also, the making of jams was taught on numerous cooking courses conducted in the first half of the 20th century all over Estonia.

\section{Future discussion}

It is difficult to compare the use of wild food plants in Estonia with that of neighbouring regions (Latvia, Finland, Russia), as they lack detailed ethnographic reviews. Still, large proportions of Rosaceae and Asteraceae make Estonia similar to other eastern European countries, e.g. Poland and Slovakia $[21,61]$. The most important detail needing attention is the low use of salads made of fresh leafy wild vegetables. That may be caused by their short-term availability in Estonia, but also by the need for hot food due to climatic conditions and the need for easier access to nutrients. In general, the large proportion of use as snacks among wild edible plants indicates a relatively good knowledge of the plants and their edibility among the population of Estonia in the past. Notable consumption of wild berries up to near-modern times reflects the fact that they are better appreciated than green vegetables.

\section{Acknowledgments}

The authors acknowledge the Governmental Research and Development programme "Estonian language and cultural memory" (EKKM09-84) for supporting the digitalisation of Estonian herbal lore. The research has been supported by ESF grant ETF9419. Many thanks to Heldur Sander for valuable references, to Tõnu Ploompuu for help with identifying species listed in ethnographic sources [Pteridium aquilinum (L.) Kuhn and Cetraria islandica (L.) Ach.], to Sarah Luczaj for language editing, to the guest editor Łukasz Łuczaj for support and inspiration and managing editor Piotr Otręba for willingness to help in improving the final version of the manuscript.

\section{References}

1. Turner NJ, Łuczaj ŁJ, Migliorini P, Pieroni A, Dreon AL, Sacchetti LE, et al. Edible and tended wild plants, traditional ecological knowledge and agroecology. Cr Rev Plant Sci. 2011;30(1-2):198-225. http://dx.doi.org/ 10.1080/07352689.2011.554492

2. Redzić $S$. Use of wild and semi-wild edible plants in nutrition and survival of people in 1430 days of siege of Sarajevo during the war in Bosnia and Herzegovina (1992-1995). Coll Antropol. 2010;34(2):551-570.

3. Pieroni A, Nebel S, Quave C, Münz H, Heinrich M. Ethnopharmacology of liakra: traditional weedy vegetables of the Arbëreshë of the Vulture area in southern Italy. J Ethnopharmacol. 2002;81(2):165-185. http://dx.doi. org/10.1016/S0378-8741(02)00052-1

4. Guarrera PM. Food medicine and minor nourishment in the folk traditions of Central Italy (Marche, Abruzzo and Latium). Fitoterapia. 2003;74(6):515-544. http://dx.doi.org/10.1016/S0367-326X(03)00122-9

5. Tardío J, Pascual H, Morales R. Wild food plants traditionally used in the province of Madrid, central Spain. Econ Bot. 2005;59(2):122-136. http:// dx.doi.org/10.1663/0013-0001(2005)059[0122:WFPTUI]2.0.CO;2

6. Pieroni A, Nebel S, Santoro RF, Heinrich M. Food for two seasons: culinary uses of non-cultivated local vegetables and mushrooms in a south Italian village. Int J Food Sci Nutr. 2005;56(4):245-272. http://dx.doi. org/10.1080/09637480500146564

7. Rivera D, Obon C, Inocencio C, Heinrich M, Verde A, Fajardo J, et al. The ethnobotanical study of local Mediterranean food plants as medicinal resources in Southern Spain. J Physiol Pharmacol. 2005;56(1 suppl):97-114.

8. Tardio J, Pardo-De-Santayana M, Morales R. Ethnobotanical review of wild edible plants in Spain. Bot J Linn Soc. 2006;152(1):27-71. http:// dx.doi.org/10.1111/j.1095-8339.2006.00549.x

9. Guarrera P, Salerno G, Caneva G. Food, flavouring and feed plant traditions in the Tyrrhenian sector of Basilicata, Italy. J Ethnobiol Ethnomed. 2006;2(1):37. http://dx.doi.org/10.1186/1746-4269-2-37

10. Pardo-de-Santayana M, Tardío J, Blanco E, Carvalho A, Lastra J, San Miguel E, et al. Traditional knowledge of wild edible plants used in the northwest of the Iberian Peninsula (Spain and Portugal): a comparative study. J Ethnobiol Ethnomed. 2007;3(1):27. http://dx.doi. org/10.1186/1746-4269-3-27

11. Leonti M, Nebel S, Rivera D, Heinrich M. Wild gathered food plants in the European Mediterranean: a comparative analysis. Econ Bot. 2006;60(2):130-142. http://dx.doi. org/10.1663/0013-0001(2006)60[130:WGFPIT]2.0.CO;2

12. Parada M, Carrió E, Vallès J. Ethnobotany of food plants in the Alt Emporda region (Catalonia, Iberian Peninsula). J Appl Bot Food Qual. 2011;84(1):11-25.

13. Menendez-Baceta G, Aceituno-Mata L, Tardío J, Reyes-García V, Pardode-Santayana M. Wild edible plants traditionally gathered in Gorbeialdea (Biscay, Basque Country). Genet Resour Crop Evol. 2011;59(7):13291347. http://dx.doi.org/10.1007/s10722-011-9760-z

14. Svanberg I, Nelson MC. Bone meal porridge, lichen soup, or mushroom bread: acceptance or rejection of food propaganda 1867-1868. In: Häkkinen A, editor. Just a sack of potatoes? Crisis experiences in European societies, past and present. Helsinki: Societas Historica Finlandiae; 1992. p. 119-147.

15. Svanberg I. The use of wild plants in the Faroe Islands 1590-1990: a contribution to Scandinavian ethnobotany. Swedish Linnean Society. 1998;1996-1997:81-130.

16. Svanberg I. Folklig botanik. Stockholm: Dialogos; 2011.

17. Redzic S. Wild edible plants and their traditional use in the human nutrition in Bosnia-Herzegovina. Ecol Food Nutr. 2006;45(3):189-232. http:// dx.doi.org/10.1080/03670240600648963

18. Pieroni A. Local plant resources in the ethnobotany of Theth, a village in the Northern Albanian Alps. Genet Resour Crop Evol. 2008;55(8):11971214. http://dx.doi.org/10.1007/s10722-008-9320-3

19. Łuczaj Ł, Kujawska M. Botanists and their childhood memories: an underutilized expert source in ethnobotanical research. Bot J Linn Soc. 2012;168(3):334-343. http://dx.doi.org/10.1111/j.1095-8339.2011.01205.x

20. Łuczaj $€$. Changes in the utilization of wild green vegetables in Poland since the 19th century: a comparison of four ethnobotanical surveys. J Ethnopharmacol. 2010;128(2):395-404. http://dx.doi.org/10.1016/j. jep.2010.01.038

21. Łuczaj $€$, Szymański WM. Wild vascular plants gathered for consumption in the Polish countryside: a review. J Ethnobiol Ethnomed. 2007;3(1):17. http://dx.doi.org/10.1186/1746-4269-3-17

22. Hupel AW. Topographische Nachrichten von Lief- und Ehstland: Nebst 
vollständigen Register über alle drey Bände. Riga: Hartknoch; 1777. (vol 2).

23. Hupel AW. Die gegenwärtige Verfassung der Rigischen und der Revalschen Statthalterschaft: Zur Ergänzung der topographischen Nachrichten von Lief- und Ehstland. Riga: Hartknoch; 1789.

24. von Luce JWL. Topographische nachrichten von der insel Oesel, in medicinischer und ökonomischer hinsicht. Riga: W. F. Häcker; 1823.

25. von Luce JWL. Nou ja abbi, kui waesus ja nälg käe on. Riga: J. H. Gressel; 1818.

26. Petri JC. Ehstland und die Ehsten, oder historisch-geographisch-statistisches Gemälde von Ehstland: Ein Seitenstück zu Merkel über die Letten. Gotha: Ettingerschen Buchhandlung; 1802. (vol 1).

27. Friebe WC. Oekonomisch-technische flora für Liefland, Ehstland und Kurland. Riga: Hartmann; 1805.

28. Wiedemann FJ, Weber E. Beschreibung der phanerogamischen Gewäschse Esth-, Liv- und Curlands mit möglichst genauer Angabe der Fundorte und der geographischen Verbreitung nebst Andeutung über den Gebrauch in medicinischer, technischer und öconomischer Beziehung. Reval: Franz Kluge; 1852.

29. Rosenplänter JH. Õppetuse katse ma Rohtudest ja pudest, mis J. H. Rosenplänter kirjutanud [Manuscript]. Tartu: Estonian Literary Museum; 1831.

30. Valk U. Eesti Sood. Tallinn: Valgus; 1988.

31. Paal J. Rare and threatened plant communitiesof Estonia. Biodivers Conserv. 1998;7(8):1027-1049. http://dx.doi.org/10.1023/A:1008857014648

32. Peterson K. Nature conservation in Estonia. Tallinn: Huma; 1994.

33. Laasimer L. Eesti NSV taimkate. Tallinn: Valgus; 1965.

34. Kukk T. Eesti taimestik. Tallinn: Estonian Academy Publishers; 1999.

35. Ööpik M, Kukk T, Kull K, Kull T. The importance of human mediation in species establishment: analysis of the alien flora of Estonia. Boreal Env Res. 2008;13:53-67.

36. Wikipedia, the free encyclopedia [Internet]. Estonia. 2012 [cited 2012 Aug 21]; Available from: http://en.wikipedia.org/wiki/Estonia

37. Kalle R, Sõukand R. Collectors of Estonian folk botanical knowledge. BJES. 2011;1:213-229.

38. Kreutzwald FR. Leivast ja leiva-jätkust. In: Tarto- ja Wõrroma kalender ehk Täht-ramat 1842 ajastaja päle, perran meie Issanda Jesusse Kristusse sündimist. Tartu: Schünmann; 1841. p. 42-51.

39. Manninen I, Linnus F. Söögid, joogid, maitsetaimed. Söögikombed. Rahvateaduslik küsimuskava. Tartu: Estonian National Musem; 1937. (Küsimusleht; vol 10).

40. Tampere H. Mahl. Tartu: Estonian National Musem; 1934. (Küsimusleht; vol 5).

41. Sion V. Seene-, marja-, pähkli- ja muu taimetoidu korjamisest. Tartu: Estonian National Musem; 1947. (Küsimusleht; vol 43).

42. Viidalepp R. Juhiseid rahvaluulekogujatele: mida ja kuidas koguda. Tartu: Estonian National Musem; 1936. (ERA Küsimuskava; vol 3).

43. Moora A. Kuidas vanasti kadakamarju kasutati. Eesti Loodus. 1984;6:378-380.

44. Moora A. Mida vanasti kasemahlast tehti. Eesti Loodus. 1982;5:298-300.

45. Moora A. Mida vanasti loodusest leivakõrvaseks korjati. Eesti Loodus. 1981;8:489-497.

46. Moora A. Marjad rahvatoidus. Eesti Loodus. 1980;8:588-592.

47. Moora A. Eesti talurahva vanem toit. 2nd ed. Tartu: Greif; 2007.

48. Kalle R, Sõukand R. Ajalooline ülevaade eestlaste looduslikest toidu- ja ravimtaimedest. In: Kusmin T, Meikar T, editors. Metsa kõrvalkasutus Eestis. Tartu: Estonian Society of Foresters; 2011. p. 29-44. (Akadeemilise Metsaseltsi Toimetised; vol 25).

49. Vilberg G. Meie kodumaa taimi rahva käsitluses. Tartu: Loodusevaatleja; 1934. (vol 1).
50. Vilberg G. Meie kodumaa taimi rahva käsitluses. Tartu: Loodusevaatleja; 1935. (vol 2).

51. Vilbaste G. Söödavaid taimi [Manuscript]. Tartu: Estonian Literary Museum; 1950.

52. Vilbaste G. Eesti taimenimetused. Tallinn: Estonian Academy of Sciences; 1993. (Emakeele Seltsi Toimetised; vol 20).

53. Kalle R, Sõukand R. Gustav Vilbaste (1885-1967) and ethnobotany in Estonia. Pioneers in European ethnobiology. In: Svanberg I, Łuczaj Ł, editors. A schoolteacher with a mission: preserving disappearing plant names. Uppsala: Royal Gustavus Adolphus Academy. In press.

54. Loorits O. Lugemispalu metsaelust ja jahindusest. 2nd ed. Tartu: Estonian Literary Museum; 2004. (Endis-Eesti Elu-Olu; vol 2).

55. Sõukand R, Kalle R. HERBA: historistlik Eesti rahvameditsiini botaaniline andmebaas [Internet]. 2008 [cited 2012 Aug 21]; Available from: http:// herba.folklore.ee

56. Sõukand R, Kalle R. Change in medical plant use in Estonian ethnomedicine: a historical comparison between 1888 and 1994. J Ethnopharmacol. 2011;135(2):251-260. http://dx.doi.org/10.1016/j.jep.2011.02.030

57. Sõukand R, Kalle R. The use of teetaimed in Estonia, 1880s-1990s. Appetite. 2012;59(2):523-530. http://dx.doi.org/10.1016/j.appet.2012.06.017

58. Kukk T, Kull T, editors. Eesti taimede levikuatlas. Tartu: Estonian University of Life Sciences; 2005.

59. Kang Y, Łuczaj ŁJ, Ye S. The highly toxic Aconitum carmichaelii Debeaux as a root vegetable in the Qinling Mountains (Shaanxi, China). Genet Resour Crop Evol. 2012;59(7):1569-1575. http://dx.doi.org/10.1007/ s10722-012-9853-3

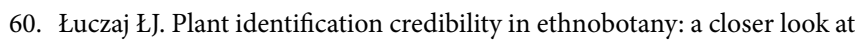
Polish ethnographic studies. J Ethnobiol Ethnomed. 2010;6(1):36. http:// dx.doi.org/10.1186/1746-4269-6-36

61. Łuczaj Ł. Ethnobotanical review of wild edible plants of Slovakia. Acta Soc Bot Pol. 2012;81(4):245-255. http://dx.doi.org/10.5586/asbp.2012.030

62. Svanberg I, Sõukand R, Łuczaj Ł, Kalle R, Zyryanova O, Dénes A, et al. Uses of tree saps in northern and eastern parts of Europe. Acta Soc Bot Pol. 2012;81(4):343-357. http://dx.doi.org/10.5586/asbp.2012.036

63. Seppel M. Näljaabi Liivi- ja Eestimaal 17. sajandist 19. sajandi alguseni. Tartu: University of Tartu Press; 2008. (Dissertationes Historiae Universitatis Tartuensis; vol 15).

64. Vahtre L. Äärmuslikkus ja äärmusetus Eesti ajaloos. In: Rohumets I, editor. Lehed ja tähed: looduse ja teaduse aastaraamat. Tallinn: Loodusajakiri; 2004. p. 99-105. (vol 2).

65. Sander H, Elliku J, Roht U. Eesti parkide ja kollektsioonide levinumate võõrlehtpuude ja -põõsaste introduktsioonist ja metsistumisest. In: Magnus R, editor. Uurimusi Eesti loodusteaduste ajaloost. Tartu: Estonian Naturalists' Society; 2008. p. 78-102. (Eesti Looduseuurijate Seltsi aastaraamat; vol 85).

66. Spuhl-Rotalia J. Kodumaa marjad. Viljandi: A. Peet; 1898.

67. Lehmann K. Kohvi-aseainete ja söögisinepi valmistamine vähemal ja suuremal mõedul. 2nd ed. Tallinn: J. Offril \& Mõte; 1909.

68. Vilbaste G. Sammalde, samblike ja vetikate nimesid. Gustav Vilbaste rahvaluulekogu (1907-1966) [Manuscript]. Tartu: Estonian Literary Museum; 1953 . (vol T3).

69. Jannau OA. Ma-rahva Koddo-Arst, ehk, lühhikenne juhhataja, kuida iggaüks mõistlik innimenne ommas maias ja perres, kui kegi haigeks saab, agga arsti ep olle sada, vöib aidata. Tartu: H. Laakmann; 1857.

70. Łuczaj ŁJ, Dumanowski J, Köhler P, Mueller-Bienick A. The use and economic value of manna grass (Glyceria) in Poland from the middle ages to the twentieth century. Hum Ecol. 2012;40:721-733. http://dx.doi. org/10.1007/s10745-012-9513-4 